\title{
Genetic Structure of Colletotrichum fructicola Associated to Apple Bitter Rot and Glomerella Leaf Spot in Southern Brazil and Uruguay
}

\author{
Mathias F. Rockenbach, Aline C. Velho, Amanda E. Gonçalves, Pedro E. Mondino, \\ Sandra M. Alaniz, and Marciel J. Stadnik
}

First, second, third, and sixth authors: Laboratory of Plant Pathology, Agricultural Science Center, Federal University of Santa Catarina, Rod. Admar Gonzaga 1346, 88034-001 Florianópolis-SC, Brazil; and fourth and fifth authors: Department of Plant Protection, Faculty of Agronomy, University of the Republic, Av. Garzón 780, CP 12900, Montevideo, Uruguay.

Accepted for publication 19 March 2016.

\begin{abstract}
Rockenbach, M. F., Velho, A. C., Gonçalves, A. E., Mondino, P. E., Alaniz, S. M., and Stadnik, M. J. 2016. Genetic structure of Colletotrichum fructicola associated to apple bitter rot and Glomerella leaf spot in Southern Brazil and Uruguay. Phytopathology 106:774-781.

Colletotrichum fructicola is the main species causing apple bitter rot (ABR) and Glomerella leaf spot (GLS) in southern Brazil, and ABR in Uruguay where GLS remains unnoticed. Thus, this work aimed to determine the genetic structure of $C$. fructicola isolates of both the countries. A total of 28 out of 31 Brazilian isolates (90.3\%) caused typical symptoms of GLS, while only 6 of 25 Uruguayan isolates (24.0\%) originating from fruits were able to infect leaves, but causing atypical symptoms. Both populations showed similar levels of Nei's gene diversity $(h=0.088$ and 0.079 , for

Brazilian and Uruguayan populations, respectively), and Bayesian cluster analysis inferred two genetic clusters correlated with the geographical origin of isolates. A principal coordinates analysis scatter plot and an unweighted pair group method with arithmetic mean-based dendrogram also grouped Brazilian and Uruguayan isolates into two groups. By pairwise comparison of nitrate-nonutilizing (nit) mutants with a proposed set of testers, all Uruguayan isolates were grouped into a unique vegetative compatibility group (namely VCG 1), while Brazilian isolates were grouped into four VCGs (VCG 1 to 4). Brazilian and Uruguayan populations of $C$. fructicola were found to be genetically distinct. Our results suggest that isolates of C. fructicola from Brazil capable of causing GLS and ABR arose independently of those from Uruguay. Possible causes leading to the evolutionary differences between populations are discussed.
\end{abstract}

Colletotrichum fructicola Prihast., L. Cai \& K.D. Hyde (2009) (Prihastuti et al. 2009) is a widely distributed fungal species that often causes plant diseases in the tropics and subtropics. Although originally described on coffee berries (Coffea arabica L.) (Prihastuti et al. 2009), it causes fruit rot and leaf spot in a broad range of host species around the world (Weir et al. 2012). Recently, C. fructicola was found to be the main species associated with apple bitter rot (ABR) and Glomerella leaf spot (GLS) in southern Brazil, and ABR in Uruguay (Velho et al. 2015), the two important diseases of apple (Malus domestica Borkh.).

$\mathrm{ABR}$ is a fruit rot disease that causes significant losses in most countries where apple is cultivated commercially (Sutton et al. 1992). Symptoms are described as initial light brown lesions in the fruit that enlarge over time becoming brown and sunken. Each lesion usually progresses into the core of the fruit in a V-shaped pattern (Sutton et al. 1992). All apple cultivars have been considered susceptible to ABR (Denardi et al. 2003).

On the other hand, the occurrence of GLS is still limited to a few regions of the world, including southeastern United States (González et al. 2006), southern Brazil (Velho et al. 2015), and China (Wang et al. 2015). In Brazil, GLS was first described in 1983 and since then it has strongly affected the local economy (Sutton and Sanhueza 1998). Despite the relative proximity to Brazilian producing areas, this disease remains unnoticed in Uruguayan orchards (Velho et al. 2015). Symptoms of GLS consist of reddish-purple leaf spots evolving to irregular necrotic lesions. Frequently, leaves turn yellow

Corresponding author: M. J. Stadnik; E-mail address: marciel.stadnik@ufsc.br

*The $\boldsymbol{e}$-Xtra logo stands for "electronic extra" and indicates that two supplementary figures are published online.

http://dx.doi.org/10.1094/PHYTO-09-15-0222-R

(C) 2016 The American Phytopathological Society and fall off (Araújo and Stadnik 2013). On fruits, it can cause small sunken lesions ( 1 to $3 \mathrm{~mm}$ ) that rarely develop into a rot (Velho et al. 2015). Apple cultivars descending from the 'Golden Delicious' group, in particular the cultivar Gala, are highly susceptible to GLS, whereas those from the 'Red Delicious' group, such as the cultivar Fuji, are completely resistant (Araújo and Stadnik 2013).

It is unknown how virulent GLS isolates arose in regions so far apart from each other such as southeastern United States, southern Brazil, and China, but not in others like Uruguay. In this context, genetic structure studies of $C$. fructicola isolates causing ABR and GLS can be helpful to understand the impact of environmental conditions, and geographical barriers influencing the distribution of populations pathogenic to fruits and leaves of apple across the world (McDermott and McDonald 1993; McDonald 1997). It can also provide information on how evolutionary forces such as selection, recombination, and gene flow can affect the persistence and viability of highly aggressive isolates, and the generation and distribution of new fungal genotypes having a potential to cause disease (McDermott and McDonald 1993; McDonald and Linde 2002).

Restriction fragment length polymorphism (RFLP)- and multilocus gene sequencing (MGS)-based analyses were already tentatively used to differentiate populations of Colletotrichum spp. causing apple diseases (González et al. 2006; Velho et al. 2015). Although MGS-based analysis could successfully differentiate Colletotrichum spp. causing ABR and GLS in Brazil and Uruguay (Velho et al. 2015), inter-simple sequences repeat (ISSR) molecular markers can be applied to resolve the genetic structure of Colletotrichum populations at a finer taxonomic level (Kumar et al. 2011; McKay et al. 2009; Rampersad 2013; Ratanacherdchai et al. 2010). The evolutionary rate within the microsatellite loci is generally higher than other genome regions, thus enabling the detection of polymorphism at individual locus (Wolfe and Liston 1998). ISSR markers also allow to differentiate populations according 
to their geographical origin or host preferences, and to estimate gene diversity, genetic differentiation, and gene flow among populations (Kumar et al. 2011; McKay et al. 2009; Rampersad 2013; Ratanacherdchai et al. 2010).

Determination of the vegetative compatibility group (VCG) is frequently used to complement the molecular-based analysis while studying the population biology of pathogens (Brooker et al. 1991; Leslie 1993). This technique is based on complementation tests using nitrate nonutilizing (nit) mutants, which are impaired to utilize nitrate as nitrogen source. When two different and compatible nit mutants anastomose, a biochemical complementation takes place in the viable heterokaryon that is detectable as the restitution of a prototrophic growth at the contact zone (Leslie 1993). Knowledge of nit mutations of testers can facilitate the determination of VCGs because complementation tests can then be directed at choosing complementary nit mutant pairs. VCGs have been previously employed to study the relationship between Colletotrichum populations from North American and Brazilian apple orchards (González et al. 2006).

The objective of this work was to determine the genetic structure of the $C$. fructicola species causing ABR and GLS in southern Brazil, and ABR in Uruguay, by means of pathogenicity tests, ISSR-based analysis, and VCGs.

\section{MATERIALS AND METHODS}

Isolates collection. Fifty-six C. fructicola isolates were selected for this study. Among these, 31 Brazilian isolates causing ABR (14) or GLS (17) were obtained from the mycological collection (MANE) of the Federal University of Santa Catarina, and 25 ABR isolates from Uruguay were provided by the culture collection (C) of the University of the Republic. Forty-six of them were previously identified by Velho et al. (2015), and 10 further GLS isolates (derived from Fraiburgo-SC, Brazil) were likewise identified.

Pathogenicity tests. The pathogenicity of isolates was tested on susceptible apple seedlings according to Araújo and Stadnik (2013). Briefly, for each isolate, three 2-month-old seedlings were inoculated by spraying the leaves with a conidial suspension $1 \times 10^{6}$ conidia/ml), and maintained for $48 \mathrm{~h}$ at $25^{\circ} \mathrm{C}$ in dark and $100 \%$ relative humidity. Subsequently, seedlings were transferred to a greenhouse $\left(25^{\circ} \mathrm{C} \pm 5^{\circ} \mathrm{C}\right)$ for further 15 days, and then assessed for incidence of symptoms of GLS. The assays were arranged in a completely randomized design.

ISSR analysis. Fungal DNA was extracted from monosporic cultures according to Gupta et al. (2013). Six isolates (MANE147, MANE34, MANE76, MANE38, C5, and C29) were used in preliminary screening to determine primers enabled to generate polymorphic and reproducible ISSR fingerprints (Table 1). PCR was performed with a $20-\mu$ l mixture containing $1 \times$ PCR buffer, $0.4 \mathrm{mM}$ dNTPs, $0.4 \mu \mathrm{M}$ primers, $1 \mathrm{U}$ of Taq DNA polymerase (SBS, Genetech Co., Ltd., China), and 5 to 15 ng of fungal genomic DNA. Standard PCR conditions were as follows: an initial denaturation step of $5 \mathrm{~min}$ at $95^{\circ} \mathrm{C}$, followed by 35 cycles of $30 \mathrm{~s}$ at $95^{\circ} \mathrm{C}, 30 \mathrm{~s}$ at $48^{\circ} \mathrm{C}$ (primers $(\mathrm{AG})_{8}$ and $(\mathrm{CT}) \mathrm{GA}_{8}$ ) or $51.5^{\circ} \mathrm{C}$ (primer $(\mathrm{CAG})_{5}$ ), and $90 \mathrm{~s}$ at $72^{\circ} \mathrm{C}$; with a final extension step of $5 \mathrm{~min}$ at $72^{\circ} \mathrm{C}$. The reactions were carried out in a thermal cycler (Labnet International, Inc.), amplicons were separated on $1.5 \%$ (wt/vol) agarose gel, stained with GelRed (Biotium, Hayward, CA), and visualized under UV light. A gene ruler 100-bp DNA ladder plus (Fermentas Life Science, Leon-Rot, Germany) was used as molecular weight marker. ISSR profiles were analyzed digitally using GelAnalyzer 2010 Software (http://www.gelanalyzer.com). Only bands of sizes between 100 and 1,500 bp that could be digitally detected were used, with the assumption that each band represented a distinct locus. Amplicons that shared the same molecular weight were considered the same locus, and data were transformed into a binary matrix $(1=$ presence, $0=$ absence $)$.

Genetic Analysis in Excel (GenAlEx) v.6.501 Software (Peakall and Smouse 2006) was used to calculate allele frequencies, bands polymorphism, and Nei's gene diversity (Nei 1973), and to generate a principal coordinates analysis (PCoA) scatter diagram. Bayesian clustering was implemented with the STRUCTURE 2.3 Software (Pritchard et al. 2000) to determine the probability of each isolate belongs to a distinct genetic cluster, assuming admixture and correlated allele frequency. Model was first run for $5 \times 10^{4}$ Markov chain Monte Carlo iterations after an initial burn-in period of $1 \times 10^{4}$ generations, from $K=1$ to $K=10$.

An unweighted pair group method with arithmetic mean (UPGMA)-based dendrogram was performed by Paleontological Statistics package (PAST) v.2.17b software (Hammer et al. 2001) to determine phylogenetic relationships between isolates. Dendrogram was generated using Dice's genetic similarity coefficient, and nodes weights were calculated with $1 \times 10^{4}$ bootstrap resamplings performed with WinBoot software (Yap and Nelson 1996).

Complementation tests and identification of nit mutants. Generation of all nit mutants was carried out according to Brooker et al. (1991). Isolates were first grown on Petri plates containing potato dextrose agar (PDA) medium supplemented with $1.5 \%$ of potassium chlorate $\left(\mathrm{KClO}_{3}\right)$ for 15 to 20 days at $25^{\circ} \mathrm{C}$ with $12 \mathrm{~h}$ photoperiod white fluorescent light. Three-millimeter-diameter agar discs with mycelia were picked up from the sectors showing dense growth, and arranged on Petri plates containing basal medium (BM) supplemented with sodium nitrate at $3.0 \mathrm{~g} /$ liter (minimum medium [MM]). Plates were incubated at $25^{\circ} \mathrm{C}$ in dark for 15 to 20 days, and nit mutants were identified by characteristic thin growth.

Complementation tests were carried out on 12-well Corning Costar cell culture plates (Sigma-Aldrich, St. Louis) containing MM. Two 3-mm-diameter agar discs containing mycelia of the generated nit mutants and testers were placed into a well about $1 \mathrm{~cm}$ away from each other. Plates were incubated at $25^{\circ} \mathrm{C}$ in dark for 15 to 20 days, and isolates that generated a white and dense heterokaryotic mycelium at the contact zone were considered compatible.

Phenotypic identification of nit testers was based on visual observation of their growth on BM supplemented with each one of the following nitrogen sources: (i) nitrate $(3.0 \mathrm{~g} / \mathrm{liter})$; (ii) nitrite ( $0.5 \mathrm{~g} /$ liter); (iii) hypoxanthine ( $0.2 \mathrm{~g} /$ liter); and (iv) uric acid ( $0.2 \mathrm{~g} /$ liter). Petri plates were kept at $25^{\circ} \mathrm{C}$ in dark for 15 to 20 days, and phenotypes were identified as described by Brooker et al. (1991).

\section{RESULTS}

Pathogenicity tests. Twenty-eight Brazilian isolates $(90.3 \%)$ caused typical GLS symptom on seedling leaves (Table 2). Symptoms started with appearance of reddish-purple irregular shaped spots at $48 \mathrm{~h}$ after inoculation that became necrotic within 7 days (Fig. 1A). Only three isolates (MANE34, MANE40, and MANE75) obtained from ABR did not cause any symptom. In contrast, six of the Uruguayan isolates (C27, C28, C29, C31, C33, and C39) infected leaves causing GLS-like symptoms. However,

TABLE 1. Characteristics of inter-simple sequence repeat primers screened

\begin{tabular}{llccc}
\hline Primer $^{\mathrm{a}}$ & \multicolumn{1}{c}{ Anchor } & Ta $\left({ }^{\circ} \mathrm{C}\right)^{\mathrm{b}}$ & Polymorphic & Reproducible \\
\hline$*(\mathrm{AG})_{8}$ & Anchorless & 48 & Yes & Yes \\
$(\mathrm{AC})_{8}$ & Anchorless & 48 & Yes & No \\
$(\mathrm{GACA})_{4}$ & Anchorless & 51.5 & No & No \\
$(\mathrm{GTG})_{5}$ & Anchorless & 51.5 & Yes & No \\
$(\mathrm{GACAC})_{3}$ & Anchorless & 48 & Yes & No \\
$(\mathrm{TGTC})_{4}$ & Anchorless & 51.5 & No & No \\
$*(\mathrm{CAG})_{5}$ & Anchorless & 51.5 & Yes & Yes \\
$\mathrm{BBD}(\mathrm{AAC})_{5}$ & $5^{\prime}$ anchor present & 53 & No & No \\
$\mathrm{BDB}(\mathrm{ACA})_{5}$ & $5^{\prime}$ anchor present & 53 & No & No \\
$(\mathrm{AG})_{8} \mathrm{TG}$ & $5^{\prime}$ anchor present & 48 & Yes & No \\
$* \mathrm{CT}(\mathrm{GA})_{8}$ & $5^{\prime}$ anchor present & 48 & Yes & Yes \\
\hline
\end{tabular}

a $*$ indicates primers selected to analyze all isolates.

b Annealing temperature. 
these isolates were less aggressive, provoking fewer and smaller reddish diffuse lesions on leaves, which appeared for more than 10 days after inoculation, but seldom progressed to necrotic symptoms (Fig. 1B and C). Leaf infection was confirmed with these isolates by re-isolating the fungus from atypical spots.

ISSR markers and bands characteristics. The three primers $\mathrm{CT}(\mathrm{GA})_{8},(\mathrm{AG})_{8}$, and $(\mathrm{CAG})_{5}$ could generate polymorphic and reproducible band patterns in the screening, and were used to analyze all isolates (Table 1). A total of 109 polymorphic bands (PB) were generated with these primers (Table 3 ). The highest number of PB were produced by $(\mathrm{AG})_{8}$ primer $(47 \mathrm{~PB})$, followed by $\mathrm{CT}(\mathrm{GA})_{8}(36$ $\mathrm{PB})$ and $(\mathrm{CAG})_{5}(26 \mathrm{~PB})$ (Supplementary Fig. S1). A high level of polymorphism was observed by considering Brazilian and Uruguayan isolates as a whole $(95.41 \%)$. However, when considered as two independent populations, the level of polymorphism was 32.11 and $22.02 \%$ for Brazilian and Uruguayan groups, respectively (Table 3).

Gene diversity. Gene diversity $(h)$, unbiased gene diversity $(u h)$, and number of different $(\mathrm{Na})$ and effective $(\mathrm{Ne})$ alleles are given in Table 3. For a whole population, $h=0.084$ and $u h=0.087$,

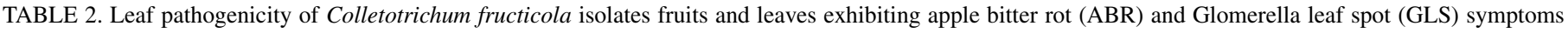
in Brazil and Uruguay

\begin{tabular}{|c|c|c|c|c|c|c|}
\hline \multirow[b]{2}{*}{ Species } & \multirow[b]{2}{*}{ Geographical origin $^{a}$} & \multicolumn{3}{|c|}{ Source } & \multirow[b]{2}{*}{ Isolate code } & \multirow[b]{2}{*}{ Pathogenicity to leaves } \\
\hline & & Cultivar & Disease & Host tissue & & \\
\hline C. fructicola & RS/Brazil & Gala & ABR & Fruit & MANE34 & - \\
\hline C. fructicola & SC/Brazil & Gala & $\mathrm{ABR}$ & Fruit & MANE35 & + \\
\hline C. fructicola & SC/Brazil & Gala & $\mathrm{ABR}$ & Fruit & MANE36 & + \\
\hline C. fructicola & SC/Brazil & Gala & $\mathrm{ABR}$ & Fruit & MANE37 & + \\
\hline C. fructicola & SC/Brazil & Gala & $\mathrm{ABR}$ & Fruit & MANE38 & + \\
\hline C. fructicola & SC/Brazil & Gala & $\mathrm{ABR}$ & Fruit & MANE39 & + \\
\hline C. fructicola & SC/Brazil & Gala & $\mathrm{ABR}$ & Fruit & MANE40 & - \\
\hline C. fructicola & SC/Brazil & Gala & $\mathrm{ABR}$ & Fruit & MANE41 & + \\
\hline C. fructicola & RS/Brazil & Gala & $\mathrm{ABR}$ & Fruit & MANE51 & + \\
\hline C. fructicola & RS/Brazil & Gala & $\mathrm{ABR}$ & Fruit & MANE54 & + \\
\hline C. fructicola & SC/Brazil & Gala & $\mathrm{ABR}$ & Fruit & MANE71 & + \\
\hline C. fructicola & SC/Brazil & Gala & ABR & Fruit & MANE73 & + \\
\hline C. fructicola & RS/Brazil & Gala & $\mathrm{ABR}$ & Fruit & MANE75 & - \\
\hline C. fructicola & SC/Brazil & Gala & $\mathrm{ABR}$ & Fruit & MANE90 & + \\
\hline C. fructicola & SC/Brazil & Gala & GLS & Leaf & MANE5 & + \\
\hline C. fructicola & SC/Brazil & Gala & GLS & Leaf & MANE7 & + \\
\hline C. fructicola & SC/Brazil & Gala & GLS & Leaf & MANE8 & + \\
\hline C. fructicola & SC/Brazil & Gala & GLS & Leaf & MANE9 & + \\
\hline C. fructicola & SC/Brazil & Gala & GLS & Leaf & MANE12 & + \\
\hline C. fructicola & SC/Brazil & Gala & GLS & Leaf & MANE16 & + \\
\hline C. fructicola & SC/Brazil & Gala & GLS & Leaf & MANE17 & + \\
\hline C. fructicola & SC/Brazil & Gala & GLS & Leaf & MANE26 & + \\
\hline C. fructicola & SC/Brazil & Gala & GLS & Leaf & MANE28 & + \\
\hline C. fructicola & SC/Brazil & Gala & GLS & Leaf & MANE31 & + \\
\hline C. fructicola & RS/Brazil & Gala & GLS & Leaf & MANE55 & + \\
\hline C. fructicola & RS/Brazil & Gala & GLS & Leaf & MANE57 & + \\
\hline C. fructicola & SC/Brazil & Gala & GLS & Leaf & MANE60 & + \\
\hline C. fructicola & SC/Brazil & Gala & GLS & Leaf & MANE70 & + \\
\hline C. fructicola & SC/Brazil & Gala & GLS & Leaf & MANE76 & + \\
\hline C. fructicola & SC/Brazil & Gala & GLS & Leaf & MANE91 & + \\
\hline C. fructicola & SC/Brazil & Gala & GLS & Leaf & MANE147 & + \\
\hline C. fructicola & MO/Uruguay & Cripps Pink & $\mathrm{ABR}$ & Fruit & $\mathrm{C} 1$ & - \\
\hline C. fructicola & MO/Uruguay & Cripps Pink & $\mathrm{ABR}$ & Fruit & $\mathrm{C} 2$ & - \\
\hline C. fructicola & MO/Uruguay & Cripps Pink & ABR & Fruit & C3 & - \\
\hline C. fructicola & MO/Uruguay & Oregon spur & ABR & Fruit & $\mathrm{C} 5$ & - \\
\hline C. fructicola & MO/Uruguay & Oregon spur & $\mathrm{ABR}$ & Fruit & $\mathrm{C} 7$ & - \\
\hline C. fructicola & MO/Uruguay & Oregon spur & ABR & Fruit & $\mathrm{C} 8$ & - \\
\hline C. fructicola & $\mathrm{CA} /$ Uruguay & Granny Smith & $\mathrm{ABR}$ & Fruit & C9 & - \\
\hline C. fructicola & CA/Uruguay & Granny Smith & $\mathrm{ABR}$ & Fruit & C10 & - \\
\hline C. fructicola & $\mathrm{CA} /$ Uruguay & Fuji & ABR & Fruit & C13 & - \\
\hline C. fructicola & $\mathrm{CA} /$ Uruguay & Fuji & ABR & Fruit & C14 & - \\
\hline C. fructicola & $\mathrm{CA} /$ Uruguay & Granny Smith & ABR & Fruit & C17 & - \\
\hline C. fructicola & $\mathrm{CA} /$ Uruguay & Cripps Pink & $\mathrm{ABR}$ & Fruit & $\mathrm{C} 22$ & - \\
\hline C. fructicola & MO/Uruguay & Granny Smith & $\mathrm{ABR}$ & Fruit & $\mathrm{C} 27$ & $+*$ \\
\hline C. fructicola & $\mathrm{CA} /$ Uruguay & Red delicious & $\mathrm{ABR}$ & Fruit & C28 & $+*$ \\
\hline C. fructicola & $\mathrm{CA} /$ Uruguay & Cripps Pink & $\mathrm{ABR}$ & Fruit & $\mathrm{C} 29$ & $+^{*}$ \\
\hline C. fructicola & $\mathrm{CA} /$ Uruguay & Cripps Pink & $\mathrm{ABR}$ & Fruit & C30 & - \\
\hline C. fructicola & $\mathrm{CA} /$ Uruguay & Cripps Pink & $\mathrm{ABR}$ & Fruit & C31 & $+^{*}$ \\
\hline C. fructicola & $\mathrm{CA} /$ Uruguay & Granny Smith & $\mathrm{ABR}$ & Fruit & C33 & $+^{*}$ \\
\hline C. fructicola & $\mathrm{CA} /$ Uruguay & Granny Smith & $\mathrm{ABR}$ & Fruit & C35 & - \\
\hline C. fructicola & $\mathrm{CA} /$ Uruguay & Granny Smith & $\mathrm{ABR}$ & Fruit & C36 & - \\
\hline C. fructicola & $\mathrm{CA} /$ Uruguay & Fuji & $\mathrm{ABR}$ & Fruit & C38 & - \\
\hline C. fructicola & $\mathrm{CA} /$ Uruguay & Fuji & $\mathrm{ABR}$ & Fruit & C39 & $+*$ \\
\hline C. fructicola & $\mathrm{CA} /$ Uruguay & Fuji & $\mathrm{ABR}$ & Fruit & $\mathrm{C} 40$ & - \\
\hline C. fructicola & $\mathrm{CA} /$ Uruguay & Fuji & $\mathrm{ABR}$ & Fruit & C41 & - \\
\hline C. fructicola & $\mathrm{CA} /$ Uruguay & Fuji & $\mathrm{ABR}$ & Fruit & $\mathrm{C} 42$ & - \\
\hline
\end{tabular}

a RS, Rio Grande do Sul; SC, Santa Catarina; MO, Montevideo; CA, Canelones.

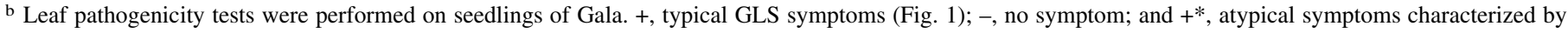
reddish diffuse lesions with longer incubation period (10 days) and seldom necrotizing of leaves (Fig. 1). 
and when considering two populations, $h=0.088$ and $u h=0.091$ were estimated for Brazilian population, and $h=0.079$ and $u h=$ 0.082 for Uruguayan population. Number of different and effective alleles were $N a=0.734$ and $N e=1.139$ for a whole population, and considering two populations, $\mathrm{Na}=0.835$ and $\mathrm{Ne}=1.141$ were estimated for Brazilian population, and $\mathrm{Na}=0.633$ and $\mathrm{Ne}=1.136$ for Uruguayan population.

Population stratification. Plot of ancestry assignment resulted in grouping Brazilian and Uruguayan isolates of $C$. fructicola into two distinct genetic clusters $(P>0.8)$ (Fig. 2). The individual's estimated membership proportion of Brazilian and Uruguayan isolates to each group were 55.4 and $44.6 \%$, respectively. PCoA scatter plot also distributed Brazilian and Uruguayan isolates forming two independent groups, which showed a clear correlation with the geographical origin instead of host tissue preference (Fig. 3).

Phylogenetics relationships. UPGMA-based dendrogram grouped Brazilian and Uruguayan populations separately with $100 \%$ of BS value, and shared up to $78 \%$ of genetic similarity (Fig. 4). Moreover, Uruguayan and Brazilian isolates shared up to 80 and $85 \%$ of genetic similarity, respectively, and no internal node within each group was found to be significant when bootstrap analysis was performed.

Vegetative compatibility grouping. Vegetative complementation grouping of isolates with the proposed set of testers allowed the classification of $55(98.2 \%)$ of the 56 isolates into four VCGs named VCG 1, 2, 3, and 4 (Fig. 4). All Uruguayan and two Brazilian isolates (MANE39 and MANE76) were included in VCG 1. On the other hand, 18 Brazilian isolates were included in VCG 2, seven in VCG 3, and three in VCG 4. One Brazilian isolate (MANE37) did not grow in $\mathrm{PDA} / \mathrm{KClO}_{3}$ mutagenic medium, and therefore, it was not possible to produce its nit mutants (Fig. 4).

Nit mutant phenotypes identification. Three types of nit mutants (nit1, nit2, and nit3) were identified for the set of testers used in the complementation assays (Table 4). Thus, pairs of testers representing VCG 1,2, and 3 were identified as nitl and nit3; and VCG 4 as nit2 and nit3 (Supplementary Fig. S2).

\section{DISCUSSION}

ISSR analysis has been successfully employed in a number of studies on genetic diversity involving Colletotrichum spp. from different hosts (Kumar et al. 2011; McKay et al. 2009; Rampersad 2013). In our study, it allowed a separation of isolates of $C$. fructicola from southern Brazil and Uruguay, but not according to the disease that they preferentially cause. Brazilian isolates were grouped into a unique genetic cluster, and the majority caused typical GLS symptoms. On the other hand, all Uruguayan isolates were grouped into another cluster and only a few were able to infect and cause symptoms on leaves, but not typical ones.

Differences in the ability to cause GLS observed between Uruguayan and Brazilian populations may have been a result of growing different cultivars in both countries for many years. Whereas the Gala (highly susceptible to GLS) is the main cultivar produced in Brazil, in Uruguay there is a predominance of those descending from 'Delicious' group such as 'Red Delicious' (53\% of total production) (MGAP 2015), which are considered to be resistant to disease (Araújo and Stadnik 2013). Thus, it is possible to assume that susceptible apple Gala, but also others descending from 'Golden Delicious' such as 'Cripps Pink', could act as a selection force on fruit-rotting populations of C. fructicola favoring the emergence of leaf disease, namely GLS (González et al. 2006; Velho et al. 2015; Wang et al. 2015). Interestingly, although most Uruguayan isolates did not cause typical GLS symptoms on apple leaves, a few of them could infect plants and provoke spots resembling the initial stages of disease and with a longer incubation period, but seldom resulting in leaf tissue necrotization (Velho et al. 2015). This observation suggests that such Uruguayan leafinfecting isolates of $C$. fructicola are in a transitional evolutionary phase, because they are not well adapted to leaf tissue colonization yet (McDonald and Linde 2002).

TABLE 3. Band characteristics and gene diversity inferred from inter-simple sequence repeat profiles of Brazilian and Uruguayan isolates of Colletotrichum fructicola causing apple bitter rot and Glomerella leaf spot on apple

\begin{tabular}{lccc}
\hline Index & Overall & $\begin{array}{c}\text { Brazilian } \\
\text { population }\end{array}$ & $\begin{array}{c}\text { Uruguayan } \\
\text { population }\end{array}$ \\
\hline Number of isolates & 56 & 31 & 25 \\
Polymorphic bands & 109 & 56 & 53 \\
Bands (frequency $>5 \%)$ & 72 & 55 & 44 \\
Private bands & 109 & 12 & 1 \\
Local bands $(<50 \%)$ & 0 & 0 & 0 \\
Mean $N a^{\text {a }}$ & 0.734 & 0.835 & 0.633 \\
SE $N a$ & 0.058 & 0.085 & 0.079 \\
Mean $N e^{\text {b }}$ & 1.139 & 1.141 & 1.136 \\
SE $N e$ & 0.019 & 0.026 & 0.028 \\
Mean $h^{\text {c }}$ & 0.084 & 0.088 & 0.079 \\
SE of Mean $h$ & 0.011 & 0.015 & 0.015 \\
Mean $u h^{\text {d }}$ & 0.087 & 0.091 & 0.082 \\
SE of Mean $u h$ & 0.011 & 0.015 & 0.016 \\
Polymorphism $(\%)$ & 95.41 & 32.11 & 22.02 \\
\hline
\end{tabular}

a $\mathrm{Na}$ : number of different alleles.

b $N e$ : number of effective alleles $\left(N e=1 /\left(p^{2}+q^{2}\right)\right.$, where $p=$ band frequency and $q=1-p$, for haploid binary data).

c $h$ : gene diversity, where $h=1-\left(p^{2}+q^{2}\right)$.

d $u h$ : unbiased gene diversity, where $u h=[N /(N-1)] \times h$.
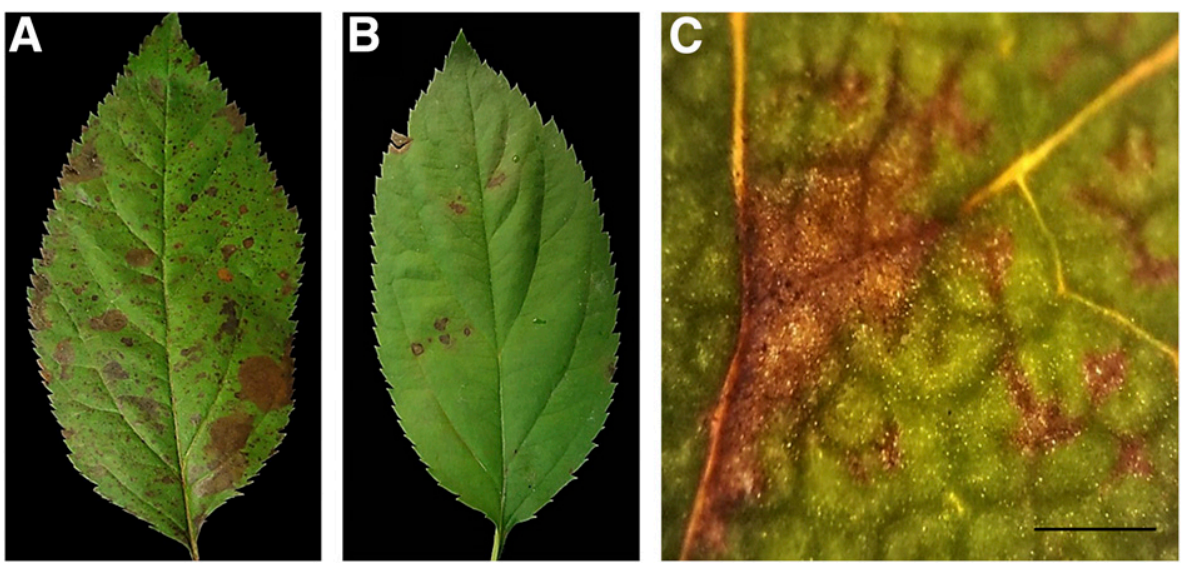

Fig. 1. A, Typical and B and C, atypical Glomerella leaf spot symptoms caused by Brazilian leaf-infecting isolate MANE147 (A), and Uruguayan fruit-infecting isolate $\mathrm{C} 29(\mathbf{B}$ and $\mathbf{C})$ of Colletotrichum fructicola, respectively. $\mathbf{C}$, Bar $=1 \mathrm{~mm}$. 
Geographical barrier separating orchards of Brazil and Uruguay may also have played an important role to promote and to maintain evolutionary divergences between Brazilian and Uruguayan populations of $C$. fructicola. It is known that geographical barriers associated with differences in environmental conditions can promote evolutionary divergences by regional or host-specific adaptation (Cannon et al. 2008; Doyle et al. 2013). Therefore, unique pathogenic genotypes tend to occupy particular geographic areas as a result of host coevolution and local adaptation to climatic conditions (Cannon et al. 2008). Brazilian and Uruguayan apple producing areas are separated by more than $1,000 \mathrm{~km}$ and climate conditions differ greatly between them. Climate conditions in southern Brazil seems to be more favorable to GLS disease occurrence (Velho et al. 2015), allowing several cycles of asexual reproduction during summer season. In this situation, recent successful mutations would be able to spread rapidly in a permissive environment (Peischl and Kirkpatrick 2012), and consequently, the chances of new fungus genotypes to survive would be higher.

Reproductive strategies can have great impact on population biology of fungal pathogens, which can show sexual and asexual reproduction simultaneously. Asexual reproduction leads to clonal lineages that tend to have lower gene diversity, and may exhibit a limited spectrum of virulence (Milgroom 1996). It is important to note that ABR isolates exclusively form acervuli on fruits. In contrast, GLS isolates can, besides acervuli, occasionally form perithecia on leaves, thus indicating the occurrence of sexual reproduction and generation of genetic variability (González et al. 2006; Velho et al. 2015). Sexual reproduction creates many new genotypes, and allows virulence genes to be recombined into different genetic backgrounds (McDonald 1997; Milgroom 1996). Hence, it would be interesting for future research to study which nutritional and genetics factors, as well as environmental conditions, stimulate the development of perithecia on leaves.

Brazilian and Uruguayan populations of $C$. fructicola showed marked differences in terms of vegetative compatibility. Interestingly, all Uruguayan isolates were grouped into only one VCG (VCG 1) similar to ISSR-based grouping, suggesting that they derived from a common population (Leslie 1993). On the other hand, four VCGs were determined for the Brazilian population, each one including both ABR and GLS isolates. Thus, it is possible
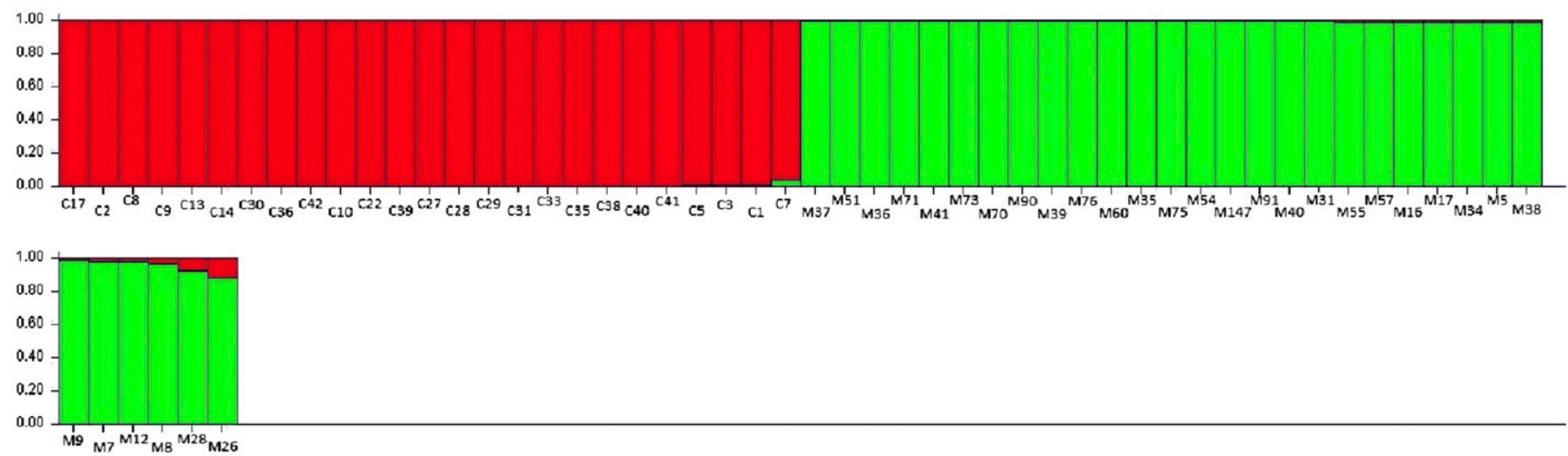

Fig. 2. Plot of ancestry estimates. Each individual is represented by a single vertical column, which is partitioned into K shaded segments that represent individual's estimated membership fraction, in each of the $\mathrm{K}$ inferred clusters. Dark gray/red and light gray/green bars correspond to Uruguayan (C) and Brazilian (M) collection isolates of Colletotrichum fructicola, respectively.

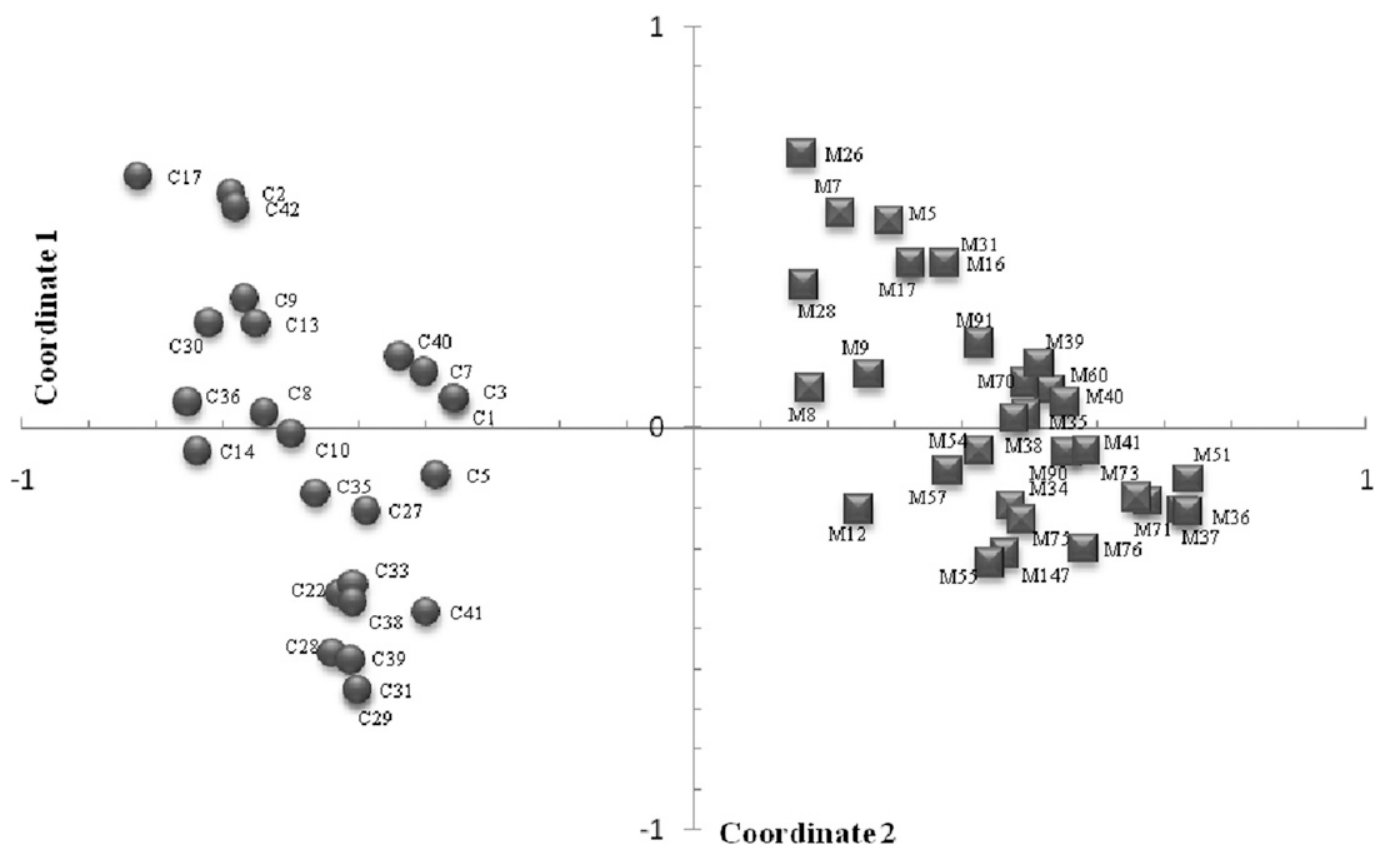

Fig. 3. Principal coordinates analysis (PCoA) scatter diagram showing the distribution of Brazilian (M) and Uruguayan (C) collection isolates of Colletotrichum fructicola, causing apple bitter rot and Glomerella leaf spot on apple. 
to argue that GLS isolates of each VCG would have evolved from ABR isolates of the same VCG. In addition, the fact that VCG 2, VCG 3, and VCG 4 were found only in Brazil suggests that they arose independently of Uruguayan population. Similarly, González et al. (2006) reported that isolates causing GLS in the United States and Brazil belonged to distinct VCGs and concluded that they emerged independently. However, we showed that Brazilian isolates MANE76 and MANE39 belonged to VCG 1, but according to ISSR-based grouping they were not different from other Brazilian isolates. Accordingly, Velho et al. (2015) through

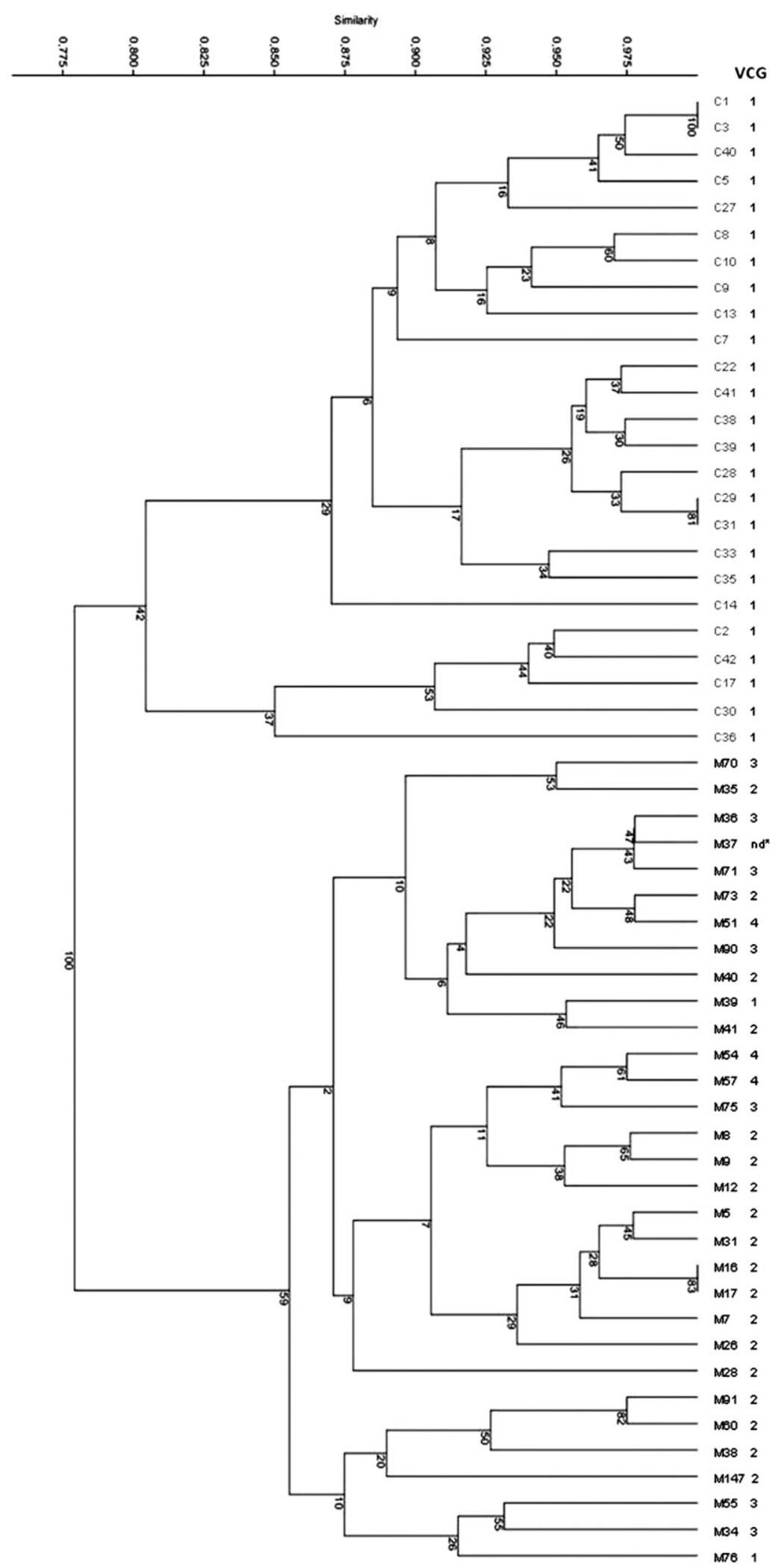

Fig. 4. Unweighted pair group method with arithmetic mean dendrogram showing the optimal tree, and vegetative compatibility group (VCG) determined for each isolate of Colletotrichum fructicola. Tree is drawn to scale, and branch lengths represent DICE's genetic similarity coefficient between groups. Bootstrap scores are shown at nodes. Correlation coefficient $=0.7763 ; *$ nd indicates not determined. 


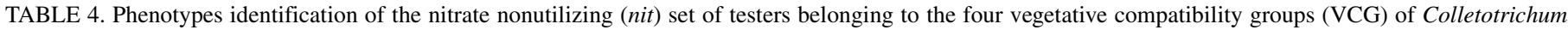
fructicola, according to growth into four nitrogen sources (nitrate, nitrite, hypoxanthine, and uric acid) ${ }^{\mathrm{a}}$

\begin{tabular}{|c|c|c|c|c|c|c|c|c|c|}
\hline \multirow[b]{2}{*}{ Species } & \multirow[b]{2}{*}{ Isolate designation } & \multirow[b]{2}{*}{ VCG } & \multirow[b]{2}{*}{ Geographical origin } & \multirow[b]{2}{*}{ Host tissue } & \multicolumn{4}{|c|}{ Nitrogen sources } & \multirow[b]{2}{*}{ Nit mutants } \\
\hline & & & & & Nitrate & Nitrite & Hypoxanthine & Uric acid & \\
\hline C. fructicola & $\mathrm{C} 29$ & 1 & CA/Uruguay & Fruit & $-1-$ & $-1+$ & $+/+$ & $+/+$ & nit3/nit1 \\
\hline C. fructicola & BR1 & 2 & SC/Brazil & Leaf & $-1-$ & $-1+$ & $+/+$ & $+/+$ & nit3/nit1 \\
\hline C. fructicola & MANE55 & 3 & RS/Brazil & Leaf & $-1-$ & $+/-$ & $+/+$ & $+/+$ & nit1/nit3 \\
\hline C. fructicola & MANE54 & 4 & RS/Brazil & Fruit & $-1-$ & $-1-$ & $-1+$ & $+/+$ & nit $2 /$ nit3 \\
\hline
\end{tabular}

a CA, Canelones; SC, Santa Catarina; RS, Rio Grande do Sul; -, thin growth without aerial mycelium; and +, wild-type growth phenotype.

MGS-based analysis found that two Brazilian isolates, i.e., MANE76 and MANE91, were more related to Uruguayan ones, which further strengthens the fact that there is a link between both populations.

The reasons for almost simultaneous emergence of GLS disease in distant regions of the world are still far from being uncovered. One hypothesis for the origin of Brazilian leaf-infecting isolates of C. fructicola is that they would have originated from those pathogenic to fruits. This is supported by the fact that Brazilian isolates causing ABR and GLS were grouped into the same genetic cluster, and because the four Brazilian VCGs shared isolates causing both diseases. It is highly unlikely that isolates causing ABR have been originated from isolates causing GLS, because the former disease has been known for about 150 years, while the latter for about 3 decades. In our work, we found some evidences that potentially leaf-infecting isolates already exist in Uruguayan population at a low frequency, but possibly environmental conditions and use of resistant cultivars could have hindered their spreading.

Another hypothesis for the GLS disease emergence in different countries is that the genotypes causing GLS could have disseminated from one region to the other. This seems, however, less probable since the conidia of Colletotrichum spp. are dispersed by water splashing that restricts their spread to short distances (Sutton and Shane 1983). On the other hand, it has been demonstrated that C. fructicola can survive overwinter in apple dormant buds and twigs (Crusius et al. 2002) that would allow, theoretically, its longdistance dispersal. Nevertheless, Brazilian imports of both apple fruits and propagative plant material have been, until now, made from GLS-free countries (MAPA 2015). Hence, comparing isolates from distant world regions where GLS occur will be an exciting challenge for future research.

In this study, the $C$. fructicola isolates causing ABR and GLS in southern Brazil and Uruguay were revealed to be genetically distinct. Brazilian GLS isolates were found to be more closely related to isolates causing ABR in Brazil than those in Uruguay, suggesting that they arose independently from the Uruguayan population.

\section{ACKNOWLEDGMENTS}

This research was financially supported by CAPES (Coordination for the improvement of higher education Personnel) PPCP-MERCOSUL 022/2011 grant. We thank T. B. Sutton (North Carolina State University) for sending the BR1 isolate, and P. K. Goteti for his help in proofreading of manuscript. M. J. Stadnik is a research member of the National Council and Technological Development (CNPq).

\section{LITERATURE CITED}

Araújo, L., and Stadnik, M. J. 2013. Cultivar-specific and ulvan-induced resistance of apple plants to Glomerella leaf spot are associated with enhanced activity of peroxidases. Acta Scientiarum 35:287-293.

Brooker, N. L., Leslie, J. F., and Dickman, M. B. 1991. Nitrate non-utilizing mutants of Colletotrichum and their use in studies of vegetative compatibility and genetic relatedness. Phytopathology 81:672-677.

Cannon, P. F., Buddie, A. G., and Bridge, P. D. 2008. The typification of Colletotrichum gloeosporioides. Mycotaxon 104:189-204.

Crusius, L., Forcelini, C. A., Sanhueza, R. M. V., and Fernandes, J. M. C. 2002. Epidemiology of apple leaf spot. Fitopatol. Bras. 27:65-70.
Denardi, F., Berton, O., and Spengler, M. M. 2003. Resistência genética à podridão amarga em maçãs, determinadas pela taxa de desenvolvimento da doença em frutos com e sem ferimentos. Rev. Bras. Frutic. 25:494-497.

Doyle, V. P., Oudemans, P. V., Rehner, S. A., and Litt, A. 2013. Habitat ad host indicate lineage identity in Colletotrichum gloeosporioides s.l. from wild and agricultural landscapes in North America. PLoS One 8:e62394.

González, E., Sutton, T. B., and Correl, J. C. 2006. Clarification of the etiology of Glomerella leaf spot and bitter rot of apple caused by Colletotrichum spp. based on morphology and genetic, molecular and pathogenicity tests. Phytopathology 96:982-992.

Gupta, V. K., Tuohy, M. G., and Gaur, R. 2013. Methods for high-quality DNA extraction from Fungi. Pages 403-406 in: A Laboratory Protocols in Fungal Biology: Current Methods in Fungal Biology. V. K. Gupta, M. G. Tuohy, M. Ayyachamy, K. M. Turner, and A. O’Donovan, eds. Springer, New York.

Hammer, O., Harper, D. A. T., and Ryan, P. 2001. Past: Paleontological statistics software package for education and data analysis. Pal. Electron 4:1-9.

Kumar, N., Jhang, T., Vir, S., and Sharma, T. R. 2011. Molecular and pathological characterization of characterization of Colletotrichum falcatum infecting subtropical Indian sugarcane. J. Phytopathol. 159:260-267.

Leslie, J. F. 1993. Fungal vegetative compatibility. Annu. Rev. Phytopathol. $31: 127-150$.

MAPA. 2015. Lista de Produtos Vegetais de Importação Autorizada (PVIA). Ministério da Agricultura, Pecuária e Abastecimento. Published online. http://www.agricultura.gov.br

McDermott, J. M., and McDonald, B. A. 1993. Gene flow in plant pathosystems. Annu. Rev. Phytopathol. 31:353-373.

McDonald, B. A. 1997. The population genetics of fungi: Tools and techniques. Phytopathology 87:448-453.

McDonald, B. A., and Linde, C. 2002. Pathogen population genetics, evolutionary potential and durable resistance. Annu. Rev. Phytopathol. 40: 349-379.

McKay, S. F., Freeman, S., Minz, D., Maymon, M., Sedgley, M., Collins, G. C., and Scott, E. S. 2009. Morphological, genetic, and pathogenic characterization of Colletotrichum acutatum, the cause of anthracnose of almond in Australia. Phytopathology 99:985-995.

MGAP. 2015. Estadísticas Agropecuárias. Pages 1-28 in: Encuesta fructicola de hoja caduca. Zafra 2014. A. Ramilo, ed. DIEA, Montevideo, Uruguay.

Milgroom, M. G. 1996. Recombination of multilocus structure of fungal populations. Annu. Rev. Phytopathol. 34:457-477.

Nei, M. 1973. Analysis of genetic diversity in subdivided populations. Proc. Natl. Acad. Sci. USA 70:3321-3323.

Peakall, R., and Smouse, P. E. 2006. Genalex 6: Genetic analysis in Excelpopulation genetic software for teaching and research. Mol. Ecol. Notes 6:288-295.

Peischl, S., and Kirkpatrick, M. 2012. Establishment of new mutations in changing environments. Genetics 191:895-906.

Prihastuti, H., Cai, L., Chan, H., McKenzie, E. H. C., and Hyde, K. D. 2009. Characterization of Colletotrichum species associated with coffee berries in northern Thailand. Fungal Divers. 39:89-109.

Pritchard, J. K., Stephens, M., and Donnelly, P. 2000. Inferences of population structure using multilocus genotype data. Genetics 155:945-959.

Rampersad, S. N. 2013. Genetic structure of Colletotrichum gloeosporioides sensu lato isolates infecting papaya inferred by multilocus ISSR markers. Phytopathology 103:182-189.

Ratanacherdchai, K., Wang, H.-K., Lin, F.-C., and Soytong, K. 2010. ISSR for comparison of cross-inoculation potential of Colletotrichum capsici causing chilli anthracnose. Afr. J. Microbiol. Res. 4:76-83.

Sutton, B. C., Bailey, J. A., and Jeger, M. J. 1992. The genus Glomerella and its anamorph Colletotrichum. Pages 1-26 in: Colletotrichum: Biology, Pathology and Control. J. A. Bailey and M. J. Jeger, eds. CABI, Wallingford, UK.

Sutton, T. B., and Sanhueza, R. M. V. 1998. Necrotic leaf blotch of Golden delicious-Glomerella leaf spot: A resolution of common names. Plant Dis. $82: 267-268$

Sutton, T. B., and Shane, W. W. 1983. Epidemiology of the perfect stage of Glomerella cingulata on apples. Phytopathology 73:1179-1183. 
Velho, A. C., Alaniz, S., Casanova, L., Mondino, P., and Stadnik, M. J. 2015. New insights into the characterization of Colletotrichum species associated with apple diseases in southern Brazil and Uruguay. Fungal Biol. 119: 229-244.

Wang, W., Fu, D., Zhang, R., and Sun, G. 2015. Etiology of apple leaf spot caused by Colletotrichum spp. Mycosystema 34:13-25.

Weir, B., Johnston, P. R., and Damm, U. 2012. The Colletotrichum gloeosporioides species complex. Stud. Mycol. 73:115-180.
Wolfe, A. D., and Liston, A. 1998. Contributions of PCR-based methods to plant systematics and evolutionary biology. Pages 43-86 in: Plant Molecular Systematics II. D. E. Soltis, P. S. Soltis, and J. J. Doyle, eds. Kluwer Academic Press, Dordrecht, The Netherlands.

Yap, V. L., and Nelson, R. J. 1996. WINBOOT: A program for performing bootstrap analysis of binary data to determine the confidence limits of UPGMA-based dendrograms. IRRI Discussion Pap. Ser. No. 14. International Rice Research Institute, Manila, Philippines. 